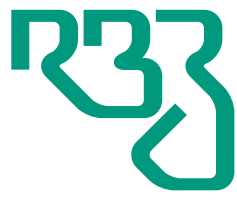

Revista Brasileira de Zootecnia

Brazilian Journal of Animal Science ISSN 1806-9290 www.rbz.org.br

\section{Serum concentration of acute phase proteins and cytokines in vaccinated pigs challenged with foot-and-mouth disease virus serotype 0}

\author{
Kyung-Woo Lee ${ }^{1}$ (iD, Kwang-Nyeong Lee ${ }^{2}$ (iD), Hyun S. Lillehoj ${ }^{3}$ (iD), \\ Jong-Hyeon Park ${ }^{2 *}$ (iD \\ Konkuk University, Gwangin-gu, Seoul, Republic of Korea. \\ Animal and Plant Quarantine Agency, Gimcheon-si, Gyeongsangbuk-do, Republic of Korea. \\ USDA, Agricultural Research Service, Beltsville Agricultural Research Center, Animal \\ Biosciences and Biotechnology Laboratory, Beltsville, MD, USA.
}

\begin{abstract}
The present study aimed to investigate the kinetics of acute phase proteins and cytokines in the serum of immunized or naive pigs following infection with a field isolate of foot-and-mouth disease (FMD) virus. Five-month-old SPF pigs were intramuscularly immunized with or without the $146 \mathrm{~S}$ antigen $(7.5 \mu \mathrm{g}$ per head) purified from FMD virus (O/Andong/SKR/2010) and were subsequently challenged 30 days post-vaccination (dpv) with FMD virus on each footpad. Serum samples were obtained at $28 \mathrm{dpv}$ and on days 1, 3, and 5 post-infection (dpi). At $28 \mathrm{dpv}$, the serum concentrations of C-reactive protein and tumor necrosis factor alpha (TNF- $\alpha$ ) were higher in the immunized pigs compared with the non-immunized control pigs. Upon challenge, the levels of all measured acute phase proteins (i.e., C-reactive protein, major acute phase protein, $\mathrm{S}$-amyloid $\mathrm{A}$, and haptoglobin) plateaued at $1 \mathrm{dpi}$ in the immunized pigs whereas they gradually increased up to $5 \mathrm{dpi}$ in the non-immunized control pigs. Levels of serum TNF- $\alpha$ remained higher in the immunized challenged pigs compared with the non-immunized challenged pigs. The serum concentration of interleukin (IL)-6 in the immunized challenged pigs was undetectable at all time points, but moderately increased upon challenge in the non-immunized challenged pigs. IL-1 $\beta$ was not detected in any of the pigs at any of the observed time points. Collectively, these findings show that the immunized pigs exhibit a rapid serum acute phase protein response following subsequent challenge with the field FMD virus compared with the non-immunized pigs.
\end{abstract}

Keywords: C-reactive protein, immune response, major acute phase protein, tumor necrosis factor alpha

\title{
Introduction
}

Foot-and-mouth disease (FMD) is a highly contagious viral disease that affects cloven-hoofed animals, including cattle, sheep, and pigs. It is caused by FMD virus, a picornavirus, the prototypical member of the genus Aphthovirus. The South East Asia (SEA) topotype is the most prevalent in Asian countries, which commonly affected cattle and swine during the FMD outbreak in the East Asian region during 2010 and 2011 (Park et al., 2014). During this outbreak, direct economic losses were estimated to be 3 billion US\$ (Sakamoto, 2012; Park et al., 2013; Saeed et al., 2015) due to the culling of 3.48 million susceptible animals. Recently, an inactivated oil-based vaccine has been developed using viruses of the O-SEA-topotype isolated in Korea and has demonstrated that vaccinated pigs (at least $7.5 \mu \mathrm{g}$ antigen/pig) are effectively protected against homologous or heterologous viral challenge (Park et al., 2014). 
It has been documented that many stressors, including disease status, can trigger the production of pro-inflammatory cytokines (i.e., interleukin [IL]-1 $\beta$, IL-6, and TNF- $\alpha$ ) from activated macrophages, which then stimulate hepatocytes to synthesize acute phase proteins (APP) (Martin de la Fuente et al., 2010). In pigs, C-reactive protein (CRP), haptoglobin, serum amyloid A (SAA), and major acute phase protein (MAP) have been identified as the major APP (Petersen et al., 2004). Moreover, APP have been used as markers of the general health status of pigs (Chen et al., 2003) and of acute FMD virus infection in cattle (Stenfeldt et al., 2011). In addition, it was found that TNF- $\alpha$ and APP (i.e., haptoglobin, ceruplasmin, fibrinogen, and SAA) were increased in FMD virus-challenged cattle compared with healthy animals (Gomez-Laguna et al., 2011; Nazifi et al., 2012). In contrast, the immunization of pigs with the FMD vaccine did not induce serum APP (i.e., alpha-1-acid glycoprotein, haptoglobin, CRP, and SAA) compared with non-immunized control pigs (Rigden et al., 2003).

Although it has been speculated that APP are systematically elevated in pigs infected with the FMD virus while remaining unaltered in pigs immunized against this virus, to date, APP have not been measured in immunized pigs following experimental infection against FMD virus. Thus, in the present study, we sought to measure the circulating patterns of selected APP and effector cytokines in immunized challenged pigs. In this short communication, four major APP (i.e., CRP, MAP, SAA, and haptoglobin) and three cytokines (i.e., IL-6, IL-1 $\beta$, and TNF- $\alpha$ ) were selected for this purpose. In addition, vaccine-induced protection against FMD virus in pigs has been reported elsewhere (Park et al., 2014). It is expected that these findings will contribute to broadening our understanding of the role of APP in monitoring the overall health status of immunized pigs upon FMD virus infection.

\section{Material and Methods}

The purification of virus particles (146S) and the infectious challenge of immunized pigs have been previously reported (Park et al., 2014). In this study, seven FMD antibody-free pigs were used to measure the serum concentration of the selected APP and cytokines upon FMD virus challenge in immunized $(n=5)$ or mock-immunized $(n=2)$ pigs. The number of the control pigs was selected based on the previous recommendation in FMD vaccine efficacy trials (Reeve et al., 2011). Briefly, five five-month-old pigs were intramuscularly immunized with the $146 \mathrm{~S}$ antigen $(7.5 \mu \mathrm{g}$ per head) purified from FMD virus (O/Andong/SKR/2010), mixed with Montanide ISA 206 VG. The pigs were subsequently challenged with FMD virus (O/Andong/SKR/2010) $\left(10^{5.0} \mathrm{TCID}_{50} / 0.1 \mathrm{~mL}\right)$ on each footpad 30 days post-vaccination (dpv). As a negative control, two pigs were immunized with PBS mixed with the adjuvant and challenged with FMD virus $30 \mathrm{dpv}$. Serum samples were obtained at $28 \mathrm{dpv}$ and on days 1,3 , and 5 post-infection (dpi) and stored at $-80^{\circ} \mathrm{C}$ until further analysis. All experimental animals were cared for according to the institutional animal management guidelines (case protocol APQA). All experiments and analyses were performed in a biosafety level 3 containment facility in Anyang,

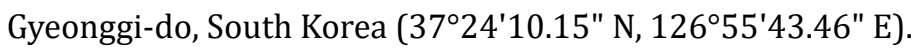

Serum APP concentrations were measured with commercially available diagnostic kits: CRP and SAA (Life Diagnostics, Inc., USA), haptoglobin (Tridelta Development Ltd., Ireland), and MAP (PigCHAMP Pro Europe S.A., Spain). For all analyses, serum samples were tested in duplicate in accordance with the manufacturer's recommendations. Prior to analysis, the serum samples were diluted as follows: 1:500 for CRP; no dilution for haptoglobin; 1:500 for SAA; and 1:1000 for pig-MAP. Serum cytokine concentrations were measured with commercially available diagnostic kits: IL-6, IL- $1 \beta$, and TNF- $\alpha$ (R\&D Systems Inc., Minneapolis, MN), and no dilutions were made. According to the manufacturer, the minimum detectable concentration (MDC) of porcine IL- 6 ranged from 0.68 to $4.30 \mathrm{pg} / \mathrm{mL}$ with a mean MDC of $2.03 \mathrm{pg} / \mathrm{mL}$; the MDC of porcine TNF- $\alpha$ ranged from 2.8 to $5.0 \mathrm{pg} / \mathrm{mL}$ with a mean $\mathrm{MDC}$ of $3.7 \mathrm{pg} / \mathrm{mL}$; and the MDC of porcine IL- $1 \beta$ ranged from 2.7 to $13.6 \mathrm{pg} / \mathrm{mL}$ with a mean MDC of $6.7 \mathrm{pg} / \mathrm{mL}$.

Individual pigs were considered an experimental unit. All values between the control and vaccine groups were analyzed with a Student's t test using the following formula:

R. Bras. Zootec., 48:e20180190, 2019 


$$
t=\frac{\left|\bar{X}_{A}-\bar{X}_{B}\right|}{\sqrt[S]{A B} \sqrt{\frac{1}{n_{A}}+\frac{1}{n_{B}}}}
$$

in which $S=$ pooled estimate of standard deviation, $\mathrm{X}=$ mean, $\mathrm{n}=$ sample size, and $\mathrm{A}$ and $\mathrm{B}=$ data from the treatment and control.

In addition, paired $t$ test was used to test for mean diferences within the treatment in serum concentrations of APP and cytokines as a function of time using the following formula:

$$
t=\frac{\bar{X}_{d}}{S d / \sqrt{n}}
$$

in which $\bar{d}=$ mean of the differences between paired observations, $S d=$ standard deviation of the difference, and $\mathrm{n}=$ number of pairs being tested.

Data were analyzed using GraphPad Prism version 6. All the results were expressed as mean \pm SD and the threshold level of statistical significance was set at $\mathrm{P}<0.05$.

\section{Results}

It was observed that CRP, but not MAP, SAA, or haptoglobin, was increased in the immunized pigs at $28 \mathrm{dpv}$ compared with the control pigs (Figure 1). At $1 \mathrm{dpi}$, the immunized pigs had higher serum concentrations of CRP $(\mathrm{P}<0.05)$, MAP $(\mathrm{P}<0.05)$, SAA $(\mathrm{P}<0.05)$, and haptoglobin $(\mathrm{P}<0.05)$ compared with the non-immunized pigs. At $3 \mathrm{dpi}$, the non-immunized pigs had higher serum concentrations of CRP $(\mathrm{P}<0.05)$ and SAA $(\mathrm{P}<0.05)$ compared with the FMD-immunized pigs. However, neither MAP nor haptoglobin differed between the immunized and the non-immunized pigs at $3 \mathrm{dpi}$. At $5 \mathrm{dpi}$, all measured APP were significantly higher in the non-immunized pigs compared with the immunized pigs. All APP, except for haptoglobin, in the immunized pigs plateaued at $1 \mathrm{dpi}$ and declined thereafter. In contrast,
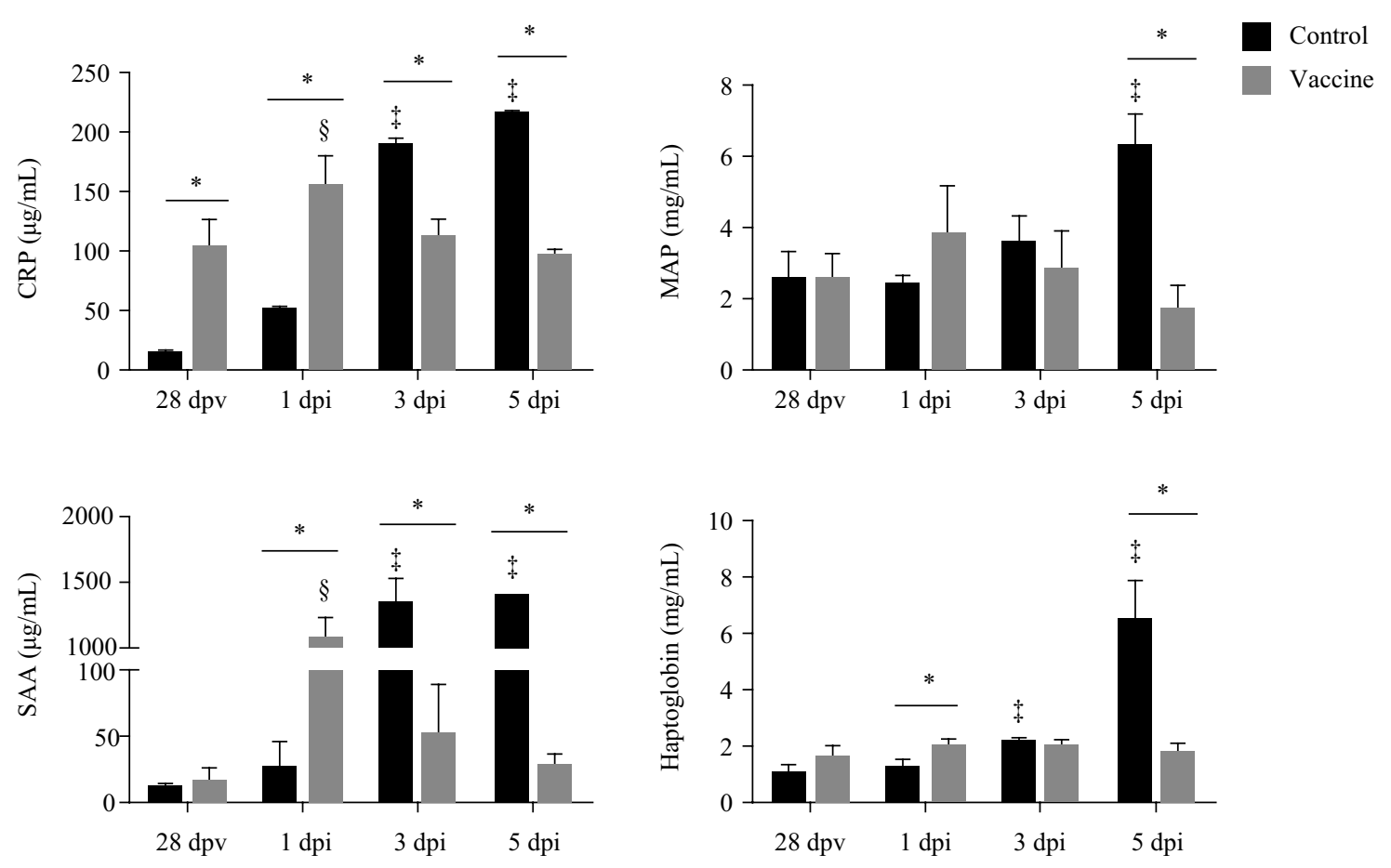

CRP - C-reactive protein; MAP - major acute phase protein; SAA - serum amyloid A; dpi - days post-infection; dpv - days post-vaccination Asterisks $(*)$ indicate significant differences between the control and vaccine groups at each time point; double daggers ( $\$$ ) indicate signficant change from $28 \mathrm{dpv}$ in the control group; section marks (§) indicate significant change from $28 \mathrm{dpv}$ in the vaccine group.

Figure 1 - Serum concentration of acute phase proteins in immunized pigs challenged against foot-and-mouth disease virus serotype 0 . 
all APP in the non-immunized challenged control pigs continued to increase until $5 \mathrm{dpi}$. Of note, serum SAA levels in the immunized pigs increased by 70.6 -fold at $1 \mathrm{dpi}$, while those in the non-immunized pigs exhibited 125.6-fold increase at $3 \mathrm{dpi}$. Similarly, serum CRP levels in the immunized pigs increased 1.5-fold at $1 \mathrm{dpi}$, while those in the non-immunized pigs exhibited 3.6-, 13.7-, and 15.7-fold increases at 1,3 , and $5 \mathrm{dpi}$, respectively.

In the serum samples, the immunized pigs exhibited higher levels of TNF- $\alpha$ compared with the non-immunized pigs at $28 \mathrm{dpv}$ (Figure 2); however, IL-6 was not detected at $28 \mathrm{dpv}$ (Table 1). The serum concentration of TNF- $\alpha$ was higher $(\mathrm{P}<0.05)$ in the immunized pigs compared with the non-immunized pigs, plateaued at $1 \mathrm{dpi}$, and then declined thereafter. IL- 6 was not detected in any of immunized pigs upon challenge against FMD virus but was detected in one of the control pigs at $1 \mathrm{dpi}$ and both pigs at 3 dpi (Table 1). IL-1 $\beta$ was not detected in any of the pigs (data not shown).

\section{Discussion}

Major stressors associated with animal production trigger the production of proinflammatory cytokines, including TNF- $\alpha$, IL- 6 , and IL- $1 \beta$, which then affect the circulating concentration of APP (Lee et al., 2016). Acute phase proteins are blood proteins primarily synthesized by hepatocytes as part of acute phase responses (Cray et al., 2009). They are known to have multiple functions, including immune modulation, protein transport, and tissue protection from damage by the inflammatory process (Cray et al., 2009). IL-6 has been established as the principle regulator of most APP, including haptoglobin and MAP (Sorensen et al., 2006). On the other hand, IL-1 $\alpha$, IL-1 $\beta$, IL-6, and TNF- $\alpha$ have also been shown to regulate a group of APP, including $\alpha$-1-acid glycoprotein, SAA, and CRP (Safi, 2012).

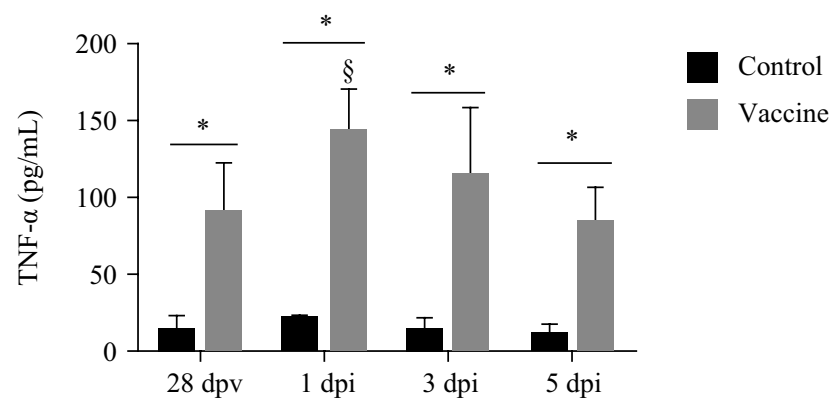

dpi - days post-infection; dpv - days post-vaccination.

Asterisks (*) indicate significant differences between the control and vaccine groups at each time point; section marks (§) indicate significant change from $28 \mathrm{dpv}$ in the vaccine group.

Figure 2 - Serum concentration of tumor necrosis factor alpha (TNF- $\alpha$; pg/mL) in immunized pigs challenged against foot-and-mouth disease virus serotype 0 .

Table 1 - Serum concentration of interleukin $6(\mathrm{pg} / \mathrm{mL})$ in immunized pigs challenged against foot-and-mouth disease (FMD) virus serotype $0^{1}$

\begin{tabular}{|c|c|c|c|c|c|}
\hline \multirow{2}{*}{ Item } & \multicolumn{2}{|c|}{ Control } & \multicolumn{2}{|c|}{ Vaccinated } & \multirow{2}{*}{ P-value } \\
\hline & Mean & SD & Mean & SD & \\
\hline $28 \mathrm{dpv}$ & ND & - & ND & - & NT \\
\hline $1 \mathrm{dpi}$ & 3.2 & - & ND & - & NT \\
\hline $3 \mathrm{dpi}$ & 33.6 & 23.5 & ND & - & NT \\
\hline $5 \mathrm{dpi}$ & 22.1 & 3.6 & ND & - & NT \\
\hline
\end{tabular}

dpv - days post-vaccination; dpi - days post-infection; ND - not detected; NT - not tested.

${ }^{1}$ Five-month-old pigs were intramuscularly immunized with or without the $146 \mathrm{~S}$ antigen, mixed with Montanide ISA 206 VG, and subsequently challeged with FMD virus $30 \mathrm{dpv}$. Serum samples were obtained at $28 \mathrm{dpv}$ and 1, 3, and $5 \mathrm{dpi}$ for interleukin 6 measurement. 
In the present study, we found that FMD virus challenge was associated with the rapid elevation of the serum concentration of APP in FMD-immunized pigs compared with that of non-immunized pigs. Upon challenge, the serum concentrations of MAP and haptoglobin in the immunized pigs briefly increased before returning to basal levels, whereas those in the non-immunized pigs gradually increased. In addition, the immunized pigs maintained substantial levels of TNF- $\alpha$, but not IL- $1 \beta$ and IL- 6 at $28 \mathrm{dpv}$ and following FMD virus challenge. Thus, these findings suggest that elevated TNF- $\alpha$ following FMD virus challenge triggered the rapid increase in CRP and SAA by hepatocytes at $1 \mathrm{dpi}$ in the immunized pigs, whereas elevated levels of IL- 6 at 3 dpi induced the rapid increase of APP in non-immunized pigs at $3 \mathrm{dpi}$ or thereafter.

Our present findings are in contrast with that of previous studies (Barnett et al., 2002; Cox et al., 2003), which reported that IL- 6 was consistently detected in both vaccinated and vaccinated challenged pigs. In a study by Cox et al. (2011), a significant association between serum IL-6 levels and FMD vaccine-specific antibody levels was observed, indicating the potential use of serum IL-6 levels as a marker of FMD vaccine efficacy. In addition, the immunization of pigs with the FMD vaccine did not induce serum APP ( $\alpha$-1-acid glycoprotein, haptoglobin, CRP, and SAA) compared with the non-immunized control pigs (Rigden et al., 2003). Thus, further study is needed to investigate the reason for the discrepancy between the present study and others regarding the level of APP and cytokines in the serum.

In livestock, APP have been used as markers of general health status (Chen et al., 2003) and acute infection (Stenfeldt et al., 2011). Moreover, it has been reported that pigs co-infected with swine influenza virus and Pasturella multocida exhibited peak levels of APP (i.e., CRP, haptoglobin, SAA, and MAP) at 2 to 3 dpi and subsequently declined (Pomorska-Mol et al., 2013). In addition, both pigs vaccinated against Aujeszky's disease (AD) displayed different patterns of serum APP upon infection compared with non-vaccinated pigs (Carpintero et al., 2007). For example, MAP and haptoglobin were elevated to a greater extent and persisted in the serum samples of non-vaccinated pigs compared with vaccinated pigs following $\mathrm{AD}$ infection. In contrast, CRP was similarly elevated but persisted in the vaccinated pigs compared with the non-vaccinated pigs following AD infection. Moreover, Chen et al. (2003) reported that due to poor growth, culled pigs displayed higher levels of HP and CRP compared with clinically healthy pigs, the former being more accurate for differentiating health status between herds. In addition, Stenfeldt et al. (2011) reported a substantial induction of SAA, haptoglobin, and type 1 interferon in serum samples upon infection, which can be used as a marker of acute FMD virus infection in cattle. Furthermore, plasma haptoglobin was found to be maintained at higher levels in cattle vaccinated against FMD compared with the non-vaccinated control group at $7 \mathrm{dpv}$ (Ferreira et al., 2016). Arthington et al. (2013) noted that in both non-vaccinated steers or heifers and those vaccinated against Mannheimia haemolytica, APP (i.e., fibrinogen or haptoglobin) were elevated and plateaued at $3 \mathrm{dpi}$.

Based on the results of our study, it appears that the dynamics of APP differ depending on the vaccination status and exhibit time-dependent kinetics regarding the production of APP.

\section{Conclusions}

In the present study, pigs immunized with or without the FMD vaccine elicited different serum APP and cytokine kinetics upon FMD virus challenge. Foot-and-mouth virus infection rapidly induces serum APP in vaccinated pigs but causes delayed APP production in non-vaccinated pigs. Based on our findings, it is likely that the four major porcine APP, when used alone or in combination, may be a useful tool for detecting FMD during the early stages of infection. It is noted that serum amyloid A elevated 126-fold at $3 \mathrm{dpi}$ and thus could be more accurate as an indicator of early FMD infection. However, their use in vaccinated pigs is limited due to either a negligible or short-lived response.

\section{Acknowledgments}

This paper was supported by Konkuk University in 2018.

R. Bras. Zootec., 48:e20180190, 2019 


\section{References}

Arthington, J. D.; Cooke, R. F.; Maddock, T. D.; Araujo, D. B.; Moriel, P.; DiLorenzo, N. and Lamb, G. C. 2013. Effects of vaccination on the acute-phase protein response and measures of performance in growing beef calves. Journal of Animal Science 91:1831-1837. https://doi.org/10.2527/jas.2012-5724

Barnett, P. V.; Cox, S. J.; Aggarwal, N.; Gerber, H. and McCullough, K. C. 2002. Further studies on the early protective responses of pigs following immunisation with high potency foot and mouth disesae vaccine. Vaccine 20:3197-3208. https://doi.org/10.1016/S0264-410X(02)00242-6

Carpintero, R.; Alonso, C.; Pineiro, M.; Iturralde, M.; Andres, M.; Le Potier, M.-F.; Madec, F.; Alava, M. A.; Pineiro, A. and Lampreave, F. 2007. Pig major acute-phase protein and apolipoprotein A-I responses correlate with the clinical course of experimentally induced African Swine Fever and Aujeszky's disease. Veterinary Research 38:741-753. https://doi. org/10.1051/vetres:2007030

Chen, H. H.; Lin, J. H.; Fung, H. P.; Ho, L. L.; Yang, P. C.; Lee, W. C.; Lee, Y. P. and Chu, R. M. 2003. Serum acute phase proteins and swine health status. Canadian Journal of Veterinary Research 67:283-290.

Cox, S. J.; Gubbins, S. and Barnett, P.V. 2011. IL-6 production following vaccination in pigs - an additional immune response parameter for assessing FMD vaccine efficacy? Vaccine 29:4704-4708. https://doi.org/10.1016/j.vaccine.2011.04.100

Cox, S. J.; Aggarwal, N.; Statham, R. J. and Barnett, P. V. 2003. Longevity of antibody and cytokine responses following vaccination with high potency emergency FMD vaccines. Vaccine 21:1336-1347. https://doi.org/10.1016/S0264410X(02)00691-6

Cray, C.; Zaias, J. and Altman, N. H. 2009. Acute phase response in animals: a review. Comparative Medicine 59:517-526.

Ferreira, L. C. L.; Cooke, R. F.; Marques, R. S.; Fernandes, H. J.; Fernandes, C. E.; Stelato, R.; Franco, G. L. and Lemos, R. A. A. 2016. Effects of vaccination against foot-and-mouth disease virus on reproductive performance of Bos indicus beef cows. Journal of Animal Science 94:401-405. https://doi.org/10.2527/jas.2015-9537

Gomez-Laguna, J.; Salguero, F. J.; Pallares, F. J.; Rodriguez-Gomez, I. M.; Barranco, I. and Carrsco, L. 2011. Acute phase proteins as biomarkers in animal health and welfare. p. 259-298. In: Acute phase proteins as early non-specific biomarkers of human and veterinary diseases. Veas, F., ed. InTech, Rijeka, Croatia.

Lee, I. K.; Kye, Y. C.; Kim, G.; Kim, H. W.; Gu, M. J.; Umboh, J.; Maaruf, K.; Kim, S. W. and Yun, C.-H. 2016. Stress, nutrition, and intestinal immune responses in pigs - a review. Asian-Australasian Journal of Animal Sciences 29:1075-1082. https://doi.org/10.5713/ajas.16.0118

Martin de la Fuente, A. J.; Carpintero, R.; Rodriguez Ferri, E. F.; Alava, M. A.; Lampreave, F. and Gutierrez Martin, C. B. 2010. Acute-phase protein resonse in pigs experimentally infected with Haemophilus parasuis. Comparative Immunology, Microbiology and Infectious Diseases 33:455-465. https://doi.org/10.1016/j.cimid.2008.11.001

Nazifi, S.; Ansari-Lari, M.; Ghafari, N.; Mohtarami, S.; Ghezelbash, A. and Tabandeh, M. R. 2012. Evaluation of sialic acids, TNF- $\alpha$, INF- $\gamma$, and acute-phase proteins in cattle infected with foot-and-mouth disease. Comparative Clinical Pathology 21:23-28. https://doi.org/10.1007/s00580-010-1059-5

Park, J. H.; Lee, K. N.; Ko, Y. J.; Kim, S. M.; Lee, H. S.; Shin, Y. K.; Sohn, H. J.; Park, J. Y.; Yeh, J. Y.; Lee, Y. H.; Kim, M. J.; Joo, Y. S.; Yoon, H.; Yoon, S. S.; Cho, I. S. and Kim, B. 2013. Control of foot-and-mouth disease during 2010-2011 epidemic, South Korea. Emerging Infectious Diseases 19:655-659. https://doi.org/10.3201/eid1904.121320

Park, J. N.; Lee, S. Y.; Chu, J. Q.; Lee, Y. J.; Kim, R. H.; Lee, K. N.; Kim, S. M.; Tark, D. S.; Kim, B. and Park, J. H. 2014. Protection to homologous and heterologous challenge in pigs immunized with vaccine against foot-and-mouth disease type 0 caused an epidemic in East Asia during 2010/2011. Vaccine 32:1882-1889. https://doi.org/10.1016/j.vaccine.2014.01.067

Petersen, H. H.; Nielsen, J. P. and Heegaard, P. M. H. 2004. Application of acute phase protein measurements in veterinary clinical chemistry. Veterinary Research 35:163-187. https://doi.org/10.1051/vetres:2004002

Pomorska-Mol, M.; Markowska-Daniel, I.; Kwit, K.; Stepniewska, K. and Pejsak, Z. 2013. C-reactive protein, haptoglobin, serum amyloid A and pig major acute phase protein response in pigs simultaneously infected with H1N1 swine influenza virus and Pasteurella multocida. BMC Veterinary Research 9:14. https://doi.org/10.1186/1746-6148-9-14

Reeve, R.; Cox, S.; Smitsaart, E.; Beascoechea, C. P.; Haas, B.; Maradei, E.; Haydon, D. T. and Barnett, P. 2011. Reducing animal experimentation in foot-and-mouth disease vaccine potency tests. Vaccine 29:5469-5473. https://doi.org/10.1016/j. vaccine.2011.05.056

Rigden, R. C.; Carrasco, C. P.; Barnett, P. V.; Summerfield, A. and McCullough, K. C. 2003. Innate immune responses following emergency vaccination against foot-and-mouth disease virus in pigs. Vaccine 21:1466-1477. https://doi.org/10.1016/ S0264-410X(02)00663-1

Saeed, A.; Kanwal, S.; Arshad, M.; Ali, M.; Shaikh, R. S. and Abubakar, M. 2015. Foot-and-mouth disease: overview of motives of disease spread and efficacy of available vaccines. Journal of Animal Science and Technology 57:10. https://doi.org/10.1186/s40781-015-0042-8

R. Bras. Zootec., 48:e20180190, 2019 
Safi, S. 2012. Acute phase protein - analysis, clinical applications and potentials. p.351-380. In: Inflamatory diseases immunopathology, clinical and pharmacological bases. Khatami, M., ed. Intech, Rijeka, Croatia.

Sakamoto, K. 2012. Mechanism of FMDV outbreaks and its control in the Asian region. Journal of Disaster Research $7: 258-263$.

Sorensen, N. S.; Tegtmeier, C.; Andresen, L. O.; Pineiro, M.; Toussaint, M. J. M.; Campbell, F. M.; Lampreave, F. and Heegaard, P. M. H. 2006. The porcine acute phase protein response to acute clinical and subclinical experimental infection with Streptococcus suis. Veterinary Immunology and Immunopathology 113:157-168. https://doi.org/10.1016/j. vetimm.2006.04.008

Stenfeldt, C.; Heegaard, P. M. H.; Stockmarr, A.; Tjornehoj, K. and Belsham, G. J. 2011. Analysis of the acute phase responses of serum amyloid A, haptoglobin and type 1 interferon in cattle experimentally infected with foot-and-mouth disease virus serotype 0. Veterinary Research 42:66. https://doi.org/10.1186/1297-9716-42-66 